\title{
Estrategias de marketing deportivo en tiempo de emergencia pandemica por el covid 19 en el destino sucre - San Vicente.Ecuador, 2021
}

\section{Sports marketing strategies in time of pandemic emergency for covid 19 in the destination sucre - San Vicente, Ecuador, 2021.}

DOI: $10.46932 / \mathrm{sfjdv2n2-033}$

Received in: january 1st, 2020

Accepted in: March 30th, 2020

\section{Frank Ángel Lemoine Quintero}

Doctorado en Ciencias Económicas,Master MBA. Universidad Laica Eloy Alfaro de Manabí, Extensión Bahía de Caráquez. Docente de la carrera de Mercadotecnia.

Dirección: Avenida Cesar Ruperti y calle Antonio Oramas, Leonidas Plaza Bahía de Caráquez, Cantón

Sucre provincia Manabí. Ecuador

Correo electrónico: flemoine1964@gmail.com

Norma Rafaela Hernández Rodríguez

Doctorado en Ciencias Económicas, Master en Dirección, Licenciada en Economía, de la Universidad de Oriente, Cuba.

Dirección: Avenida Patricio Lumumba s/n. Santiago de Cuba, Cuba

Correo electrónico: norma@uo.edu.cu

\section{Josselin Marlene Castro Robles}

Estudiante de Mercadotecnia. Universidad Laica Eloy Alfaro de Manabí, Extensión Bahía de Caráquez.

Docente de la carrera de Mercadotecnia.

Dirección: Avenida Cesar Ruperti y calle Antonio Oramas, Leonidas Plaza Bahía de Caráquez, Cantón

Sucre provincia Manabí. Ecuador

Correo electrónico: cjoselin512@gmail.com

\section{Kely Jenifer Zambrano Arteaga}

Estudiante de Mercadotecnia. Universidad Laica Eloy Alfaro de Manabí, Extensión Bahía de Caráquez.

Docente de la carrera de Mercadotecnia.

Dirección: Avenida Cesar Ruperti y calle Antonio Oramas, Leonidas Plaza Bahía de Caráquez, Cantón

Sucre provincia Manabí. Ecuador

Correo electrónico: kely_97@outlook.com

\section{RESUMEN}

La investigación tuvo como objetivo desarrollar estrategias de marketing para gerenciar productor deportivo bajo las condiciones pandémicas producida por el Covid 19 como una alternativa de reactivar la actividad turística y económica en la zona objeto estudio en el destino Sucre-San Vicente. Se realizó un estudio a nivel del estado del arte bibliográfico que aporte al estudio determinando que a través del método analítico y descriptivo se puede obtener resultados favorables a la investigación. Se analizaron los resultados del cuestionario aplicado a un segmento del mercado de la ciudad que, según muestreo aleatorio simple arrojó resultados satisfactorios, donde lo más representativo fue el nivel de aceptación la actividad deportiva como producto turístico sostenible para el destino. La matriz de ponderación muestra que los deportes playeros superan otro tipo de actividad deportiva, aunque se denota la práctica del ciclismo como una actividad de muy buena aceptación. Se definieron cuatro estrategias que permitirán gerenciar la actividad deportiva como producto turístico para la sostenibilidad del destino. 
Palabras claves: estrategias, actividad deportiva, producto turístico, sostenibilidad

\begin{abstract}
The objective of the research was to develop marketing strategies to manage sports producers under the pandemic conditions produced by Covid 19 as an alternative to reactivate the tourist and economic activity in the area under study in the Sucre-San Vicente destination. A study was carried out at the level of the state of the art bibliography that contributes to the study, determining that through the analytical and descriptive method, favorable results can be obtained for the research. The results of the questionnaire applied to a segment of the city's market were analyzed, which, according to simple random sampling, yielded satisfactory results, where the most representative was the level of acceptance of sports activities as a sustainable tourism product for the destination. The weighting matrix shows that beach sports outweigh other types of sports activities, although cycling is noted as a very well accepted activity. Four strategies were defined that will allow the management of sports activities as a tourism product for the sustainability of the destination.
\end{abstract}

Key words: strategies, sports activity, tourism product, sustainability.

\title{
1 INTRODUCCION
}

En la sociedad actual el deporte y las actividades físicas amplían su espectro de disciplinas y están en continua evolución. Podemos decir que los jóvenes además de las prácticas generalizadas de deporte federado y deporte salud, se inclinan por prácticas de deporte ligadas al turismo y por deportes de aventura y de riesgo. Si en general la sociedad justifica el no hacer deporte o no hacer más deporte por la falta de tiempo, este colectivo es uno de los que más acusa esta problemática, ya que están dedicados a sus estudios y hablando de los "maduros de la juventud", ya tienen trabajo y cargas familiares a una práctica más recreativa y dirigida a la salud, normalmente más individual. Además, el factor de mejora de la estética corporal juega un papel importante a la hora de practicar deporte o actividad física, exigencia más acentuada hacia las mujeres, pero al que el hombre está siendo implicado y muy influenciado. (INFORME JUVENTUD, 2010)

Al definir el término "producto turístico" nos estamos refiriendo a: una combinación de bienes y servicios ofertados a un precio global. De tal modo, podemos decir que un producto turístico deportivo, será aquel cuya principal motivación será la de acudir algún evento deportivo como espectador o bien, la práctica de algún deporte al realizar un viaje. El deporte se está convirtiendo en los últimos años en un elemento clave para la sociedad actual y cada vez más está más ligado al turismo, compaginando la actividad turística con la práctica deportiva. (Peláez, 2009)

En el país el turismo de deporte o aventura se ha convertido en una de las actividades convenientes, considerándose una de las causas que contribuye a la economía del país. Teniendo gran importancia para 
la adaptabilidad de esta clase de turismo alternativo, contando con lugares donde se pueda realizar. (Bohorquez, 2016)

(Morillo, 2011) Definen al producto turístico como el conjunto de bienes y servicios que se ofrecen al mercado en forma individual o en combinaciones de acuerdo a las necesidades y requerimientos de los turistas. Dichos bienes y servicios pueden estar destinados al confort y disfrute material o espiritual de los visitantes.

(Turistico, 2017) a partir de los criterios de Roberto Boullon establece que el producto turístico es algo más complejo y está conformado por toda la oferta turística, integrada, a su vez, por los servicios que suministran los elementos de la planta turística y por algunos bienes no turísticos. En otras palabras, la oferta turística son todos los bienes y servicios disponibles para el turista por un precio y en un período dado, condiciones estas que determinan su característica de transitoriedad, es decir su imposibilidad de almacenamiento.

El problema de la investigación está fundamentado en el uso inadecuado de estrategia de marketing deportivo como técnica para fortalecer la actividad deportiva bajo las condiciones del Covid 19 en el destino Sucre-San Vicente. Además, el uso inadecuado de la identificación y caracterización de los atractivos son elementos básicos para fortalecer la actividad deportiva, pero estos no son concebidos por las organizaciones gubernamentales y gobiernos GAD, por la ausencia de una planificación y proyección de estas actividades como actividad turística bajo las condiciones de emergencia pandémica.

El objetivo que se persigue con esta investigación es desarrollar estrategias de marketing para gerenciar productos en el destino Sucre-San Vicente con la finalidad de fortalecer el turismo y hacer de este una actividad sostenible en el destino.

\section{MARCO TEÓRICO}

Evolución histórica de las estrategias publicitarias en la dinamización deportiva Según (González \& Francisco, 2006) citaron a (Kotler, 2001) que podríamos definir el marketing como: "Una orientación, filosofía o sistema de pensamiento, de la dirección que sostiene que la clave para alcanzar las metas de la organización reside en averiguar las necesidades y deseos del mercado objetivo, y en adaptarse para ofrecer las satisfacciones deseadas por el mercado mejor y más eficientemente que la competencia".

Se puede entender como marketing deportivo como el conjunto de acciones y prestaciones, producidas en sentido a satisfaces las necesidades, expectativas y preferencias de los consumidos de deporte. Este es el conjunto de acciones y prestaciones se desarrolla en dos grandes áreas, o mejor en dos grandes segmentos (1) instituciones públicas y organizaciones que ofrecen servicios deportivos, desde 
dos planos: práctica deportiva, espectáculo deportivo, (2) empresas que comercializan productos para la práctica deportiva, o que proporcionan contenidos deportivos. Según (Lema, 2015).

Según Crua, 2016 el marketing deportivo ya forma parte de la esencia del deporte; vemos publicidad en las camisetas, en los campos, y ahora hasta en las redes. Las estrategias de comunicación y comercialización dentro del mundo deportivo se han convertido en un deporte en sí mismas. ¿Cuál es su meta? En primera estancia, ganar dinero, y en segunda, satisfacer las necesidades y deseos de las personas que practican deporte, creando para ellos productos y servicios destinados a hacer actividad física o relacionada con el mundo del deporte.

La estrategia que conforma parte del marketing se manifiesta constantemente en cualquier contexto social. Todos planteamos "estrategias" de algún modo cuando nos fijamos metas y reflexionamos respecto a la probabilidad de alcanzarlas y el cómo lograrlo. El lineal planifica estrategias para culminar sus estudios, una familia planifica su economía, etcétera. Por tanto, podemos decir que la estrategia es el proyecto o programa que se elabora sobre determinada base, para alcanzar el objetivo propuesto según (Collazo, 2014).

\section{MATERIAL Y MÉTODO}

Los métodos que se utilizaran en la presente investigación son el analítico y descriptivo.

Analítico: Partiendo del estudio del estado del arte (artículos, libros, revistas, etc.) que permitan desarrollar estrategias de marketing efectivas para gerenciar la actividad deportiva en el destino

Descriptivo: Se realizará un estudio referente a las actividades deportivas en la zona que se realizan eventualmente midiendo su nivel de frecuencia. Se partirá de la identificación de los atractivos para valorar las actividades deportivas que se pueden introducir.

POBLACIÓN: La población que se pretende estudiar son los habitantes de los destinos Sucre y San Vicente. De los cuales se escogerán la población activa según rango de edades superior a los 18 años e inferior a los 50 años.

Muestreo aleatorio no probabilístico: La muestra es de 50 personas de un rango de edad superior a los 18 años.

\section{RESULTADOS}

El cantón Sucre tiene como cabecera cantonal a la ciudad de Bahía de Caráquez, su nombre se debe a la Bahía formada por la desembocadura del río Chone y a los primeros pobladores que fueron los Caras. Bahía de Caráquez cuenta con un gran atractivo turístico y frente a ella está San Vicente, cruzando en lancha, gabarra o por vía terrestre y cuenta con el aeropuerto "Los Perales". De norte a sur existen 
Figura 1. Georreferenciación del destino Sucre-San Vicente

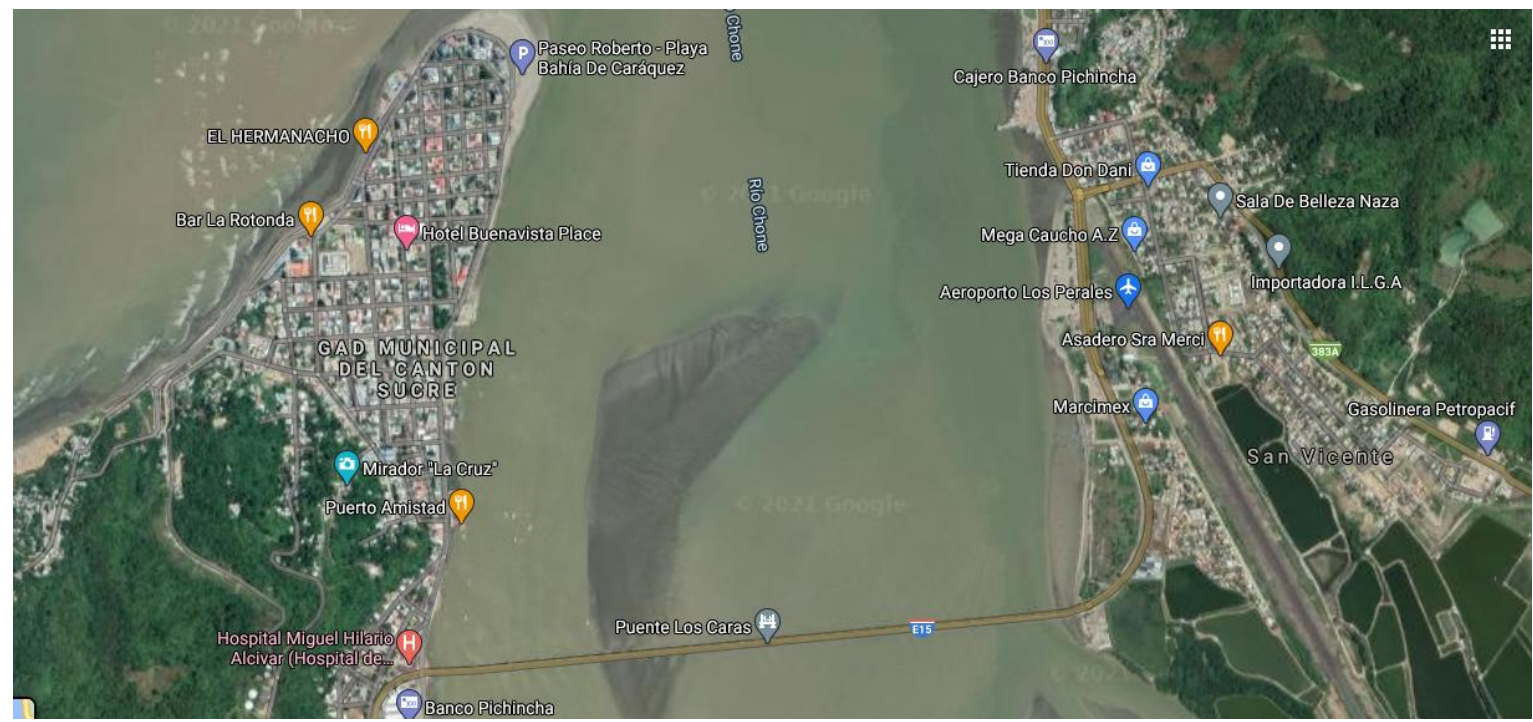

Fuente: Google maps, 2021

\subsection{RESULTADOS DE LA ENCUESTA}

Se tomó como referencia los resultados de las encuestas realizadas por el estudiante Jonathan Stalin Farías Paladines en su trabajo de titulación denominado "Estrategias publicitarias para dinamizar la actividad deportiva en la parroquia Bahía de Caráquez” con la finalidad de realizar un análisis a nivel interpretativo de estos resultados.

La encuesta conto con 10 preguntas de ellas 4 direccionada al perfil del cliente y 6 preguntas con escala de intensidad o de apreciación estructuran las opiniones bajo formas de respuesta en abanico, según la evolución o grados de un continuum de actitud, donde el valor 1 corresponde a nade de acuerdo, 2 algo en desacuerdo, 3 ni de acuerdo ni en desacuerdo, 4 completamente de acuerdo.

Referente al perfil del cliente la pregunta direccionada a géneros de ciudadanos de un total de 50 personas encuestadas se puede establecer que 30 personas que equivalen al $60 \%$ son de género masculino y 20 personas que equivalen al $40 \%$ son de género femenino. Se puede concluir que la mayor parte de las 
personas encuestadas fueron 30 hombres, mientras que las mujeres fueron 20, es decir, que el segmento al que más se enfoca en los deportes son los hombres.

La pregunta relacionada a la edad de los ciudadanos con la encuesta realizada el $40 \%$ de los investigados corresponde a 20 personas en un rango de edad de 10 a 24 años, seguido de un $30 \%$ es decir 15 personas que se encuentran en una edad de 25 a 39 años y otro $30 \%$ que compete a 15 personas que se encuentran en una edad de 40 a 69. Estos rangos de edades fueron determinado por el software REDATAM. En relación a los resultados obtenidos se puede decir que la mayor parte de los usuarios que practican deporte son personas entre el rango de 10 a 24 años, mientras que el resto de personas encuestadas están en un rango de edad entre 25 a 69 años.

Otra pregunta que arrojo resultados significativos fue referente a la actividad deportiva si contribuye a la actividad física y recreativa del sector turístico, donde de una población de 50 personas encuestadas se puede establecer que 20 personas que equivalen a un $40 \%$ están completamente de acuerdo que si se permitirá la actividad deportiva desarrollar la actividad física y deportiva dentro del sector turístico, 14 personas que equivalen a un $28 \%$ están algo de acuerdo en que si se permitirá la actividad deportiva desarrollar la actividad física y deportiva dentro del sector turístico, 6 personas que equivalen a un $12 \%$ están algo en desacuerdo en que se permitirá la actividad deportiva desarrollar la actividad física y deportiva dentro del sector turístico, 6 personas más que corresponden a un 12\% no están de acuerdo en que se permitirá la actividad deportiva desarrollar la actividad física y deportiva dentro del sector turístico y 4 personas que semejan a un $8 \%$ no están ni de acuerdo ni en desacuerdo en que se permitirá la actividad deportiva desarrollar la actividad física y deportiva dentro del sector turístico.

La pregunta referida a los atractivos turísticos existentes en el cantón si contribuirán a dinamizar la actividad deportiva de una población, respondieron 20 personas que equivalen a un $40 \%$ están algo de acuerdo en que los atractivos turísticos existentes en el cantón contribuirán a dinamizar la actividad deportiva, 15 personas que representan un 30\% están completamente de acuerdo, 6 personas que representan un $12 \%$ respondieron en estar ni de acuerdo en que los atractivos turísticos existentes en el cantón contribuirán a dinamizar la actividad deportiva, 5 personas más que equivalen a un $10 \%$ están algo en desacuerdo en que los atractivos turísticos existentes en el cantón contribuirán a dinamizar la actividad deportiva, además 4 personas que figuran un $8 \%$ no están ni en desacuerdo en que los atractivos turísticos existentes en el cantón contribuirán a dinamizar la actividad deportiva.

Desarrollar una imagen a la actividad deportiva permitirá fortalecer la actividad turística en la ciudad se puede establecer que 18 personas que equivalen a un $36 \%$ están completamente de acuerdo en desarrollar una imagen que representa a la actividad deportiva para fortalecer la actividad turística en la ciudad, un $24 \%$ están algo de acuerdo en desarrollar una imagen a la actividad deportiva permitirá 
fortalecer la actividad turística en la ciudad, un $20 \%$ no están de acuerdo en desarrollar una imagen a la actividad deportiva permitirá fortalecer la actividad turística en la ciudad, un $10 \%$ están algo en desacuerdo en desarrollar una imagen a la actividad deportiva permitirá fortalecer la actividad turística en la ciudad y otro 10\% no están ni en desacuerdo en desarrollar una imagen a la actividad deportiva permitirá fortalecer la actividad turística en la ciudad.

Referente a las estrategias publicitarias y su contribución al desarrollo de la actividad deportiva y turística de la zona un $44 \%$ respondieron estar completamente de acuerdo en que estas contribuyen al desarrollo en la actividad deportiva y turística de la zona, un 32\% están algo de acuerdo, un 10\% no están de acuerdo en que las estrategias publicitarias contribuyen al desarrollo en la actividad deportiva y turística de la zona, un $8 \%$ están algo de acuerdo y un $6 \%$ están algo en desacuerdo de que estas aporten al desarrollo en la actividad deportiva y turística de la zona.

Con quién prefieres realizar actividad deportiva o física se puede establecer como indica los resultados que 15 personas que equivalen a un $30 \%$ prefieren realizar actividad física o deportiva con cualquiera, 14 personas que equivalen a un $28 \%$ eligen realizar actividades físicas o deportivas con familiares, 10 personas que equivalen a un $20 \%$ optan las actividades físicas o deportivas con amistades, 6 personas más que equivalen a un $12 \%$ distinguen por las actividades físicas o deportivas con pareja y otras 5 personas que equivalen a un $10 \%$ escogen las actividades físicas o deportivas.

Gráfico 1. Particularidades de participación en actividad deportiva

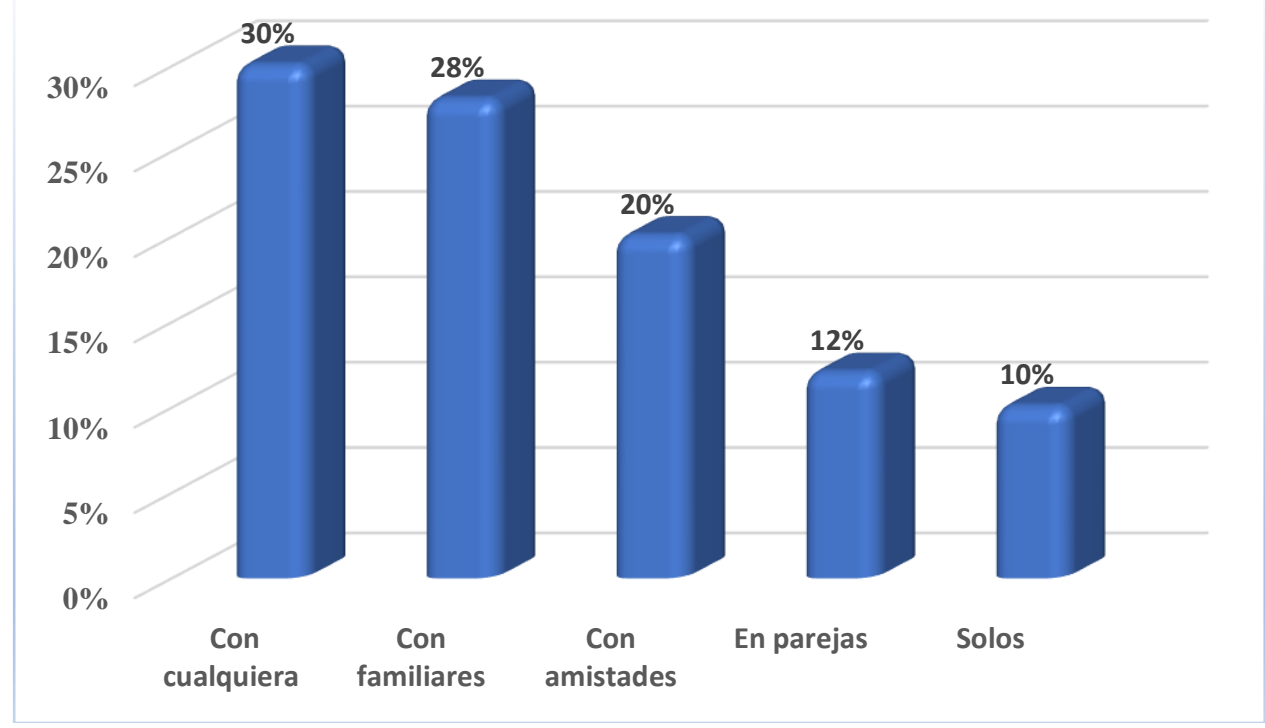

Fuente: Autores, 2021

De los deportes extremos a seleccionar en la pregunta la tabla de frecuencia que 9 personas que equivalen a un $18 \%$ les gustaría practicar de deportes extremos la motocross, 9 personas que equivalen a un $18 \%$ les gustaría practicar de deportes extremos el ciclismo de montaña, 8 personas que equivalen a 
un $16 \%$ les gustaría practicar de deportes extremos el parapente, 7 personas que equivalen a un $14 \%$ les gustaría practicar de deporte extremos el surf, 7 personas más que equivalen a un 14\% les gustaría practicar de deportes extremos el montañismo o el alpinismo y otras 7 personas más que equivalen a otro $14 \%$ les gustaría practicar de deportes extremos el buceo deportivo y por ultimo 3 personas que equivalen a un $6 \%$ les gustaría practicar como deportes extremo el kayak.

Estas respuestas han denotado que existe una población que reconocer la actividad deportiva como una forma de fortalecer la actividad turística en la zona partiendo de los atractivos existente, por la ubicación del destino y como estrategia sostenible para posicionar el destino en un mercado nacional e internacional.

\subsection{ESTUDIO DE LA ACTIVIDAD DEPORTIVA}

Desde hace bastante tiempo se presumía que la actividad física podría tener relación con una mejoría de los procesos cognitivos que tienen su origen en el cerebro, pero gracias a una serie de estudios desarrollados por la Universidad de Illinois, en los Estados Unidos, esta suposición terminó siendo una comprobación empírica que arrojó como resultado que, efectivamente, a mayor actividad aeróbica, menor degeneración neuronal. (Ramires R, 2004)

Estas actividades, aseguran los autores, surgen con el advenimiento de la sociedad postindustrial, de la mano de la lógica mercantil y bajo las estrategias comerciales. La propia promoción de las actividades, por parte de las empresas que las desarrollan, va dirigida a demostrar a las personas que necesitan evadirse de la rutina y del estrés diario y así dejarse invadir por la emoción del riesgo y aventura. De tal manera, que es habitual que el espacio natural sea en cierta forma "invadido" por una serie de infraestructuras de las empresas que desarrollan estas actividades.

La matriz de ponderación permitirá a partir de criterio de experto teniendo conocimiento de las actividades deportivas más frecuente en el destino cuales de estas tienen una estacionalidad más prolongada en el tiempo, cual una mediana o baja estacionalidad. La ponderación será por el nivel de impacto que tiene la misma en el destino, la calificación será de 1 a 5, 1 la puntuación más baja determinada por el nivel de participación de personas o deportistas en diferentes temporadas del año y 5 la de mayor puntuación. Participaran 8 expertos, dos especialistas en marketing, dos en turismo, dos representantes barriales y dos estudiantes de la carrera de marketing, cuyos resultados se muestran en la siguiente tabla. 
Tabla 1. Matriz de ponderación

\begin{tabular}{|l|c|c|c|}
\hline ACTIVIDAD DEPORTIVA & PONDERACION & CLASIFICACIÓN & RESULTADO \\
\hline Surf & 0.097 & 4.5 & 0.44 \\
\hline Futbol playero & 0.22 & 4 & 0.88 \\
\hline Pesca deportiva & 0.086 & 2 & 0.17 \\
\hline Balsaje & 0.065 & 1.5 & 0.10 \\
\hline Senderismo & 0.11 & 4.7 & 0.52 \\
\hline Ciclismo & 0.13 & 4.6 & 0.60 \\
\hline Vóleibol & 0.12 & 3.5 & 0.42 \\
\hline Kayak & 0.096 & 2.3 & 0.22 \\
\hline Camping & 0.076 & 1.25 & 0.10 \\
\hline TOTAL & 1 & & $\mathbf{3 . 4 4}$ \\
\hline
\end{tabular}

Fuentes: Autores, 2021

De la encuesta aplicada a el segmento de mercado de la localidad arrojo resultados alentadores, debido a que la actividad deportiva la consideran como una alternativa para desarrollar productos turísticos de diferentes tipos y para diferentes segmentos de mercado.

Dentro de los resultados de la encuesta es notorio como la población hace preferencias por los deportes extremos, esto es debido a la influencia de turistas internacionales que buscan un turismo de aventura teniendo presente que un $36 \%$ de los encuestados consideraron que hay que desarrollar una imagen que identifique esta actividad con la finalidad de posicionarla en el mercado, donde fue representativo que el 36\% también consideraron que esta actividad puede potencializar el destino y ser parte de la reactivación económica. Por lo que existen resultados que puntualizan que se necesita gerenciar bien estas actividades deportivas teniendo presente una estacionalidad de la demanda de acuerdo a las estaciones climáticas vigentes en el territorio.

La matriz de ponderación muestra que de las actividades deportivas que en la actualidad se realizan como deportes en el destino existen un nivel de representatividad de algunas más que otras por los moradores o actores de ambas ciudades que desde sus iniciativas han creado áreas y eventos competitivos como parte de su recreación. Estos deportes más representativos son el futbol playero y el Vóleibol, aunque existen otros que tienen el mismo nivel de representatividad o aceptación, pero por su particularidad en los recursos que deben de tener no son aún tan representativo en la zona.

Respecto a las estrategias para gerenciar estas actividades deportiva para fortalecer el turismo en el destino hay que fundamentar dos momentos, los mismos están dado por un análisis de correlación entre la encuesta aplicada al segmento de mercado de la ciudad de Bahía de Caráquez y sobre el estudio realizado por expertos sobre las actividades turísticas que se realizan con frecuencia en determinados 
atractivos existente en la zona, para esto se muestra una tabla de proyección de actividad deportiva, la cual se muestra a continuación.

\begin{tabular}{|l|l|l|}
\hline \multicolumn{2}{|c|}{ Tabla 2. Proyecciones de las actividades deportivas en el destino } \\
\hline $\begin{array}{l}\text { ACTIVIDAD } \\
\text { DEPORTIVA }\end{array}$ & ATRACTIVO & $\begin{array}{l}\text { TIEMPO/ESTACIONALIDAD } \\
\text { DEPORTIVA }\end{array}$ \\
\hline \multicolumn{1}{|c|}{ Surf } & Playa & Meses: Mayo y Septiembre \\
\hline Futbol playero & Playa & Todo el año \\
\hline Pesca deportiva & Playa & Según tabla de mareas \\
\hline Balsaje & Playa, estuario del rio Chone & Verano: mayo a junio \\
\hline Senderismo & $\begin{array}{l}\text { Reserva Ecológica Cerro } \\
\text { Seco }\end{array}$ & $\begin{array}{l}\text { Meses : Mayo a Junio y de Septiembre } \\
\text { a Diciembre }\end{array}$ \\
\hline Ciclismo & Cerro Seco, ciclopaseos & Verano: mayo a junio \\
\hline Vóleibol & Playa del litoral & Todo el año \\
\hline Kayak & Playa y estuario rio Chone & Verano: mayo a junio \\
\hline Camping & Bosques(Sitios naturales) & Verano: mayo a junio \\
\hline
\end{tabular}

Fuentes: Autores, 2021

Dentro de las actividades antes mencionadas y sus proyecciones es importante argumentar sobre el desarrollo de una Rutas Ciclistas desde el criterio de especialista es una actividad deportiva que toma fuerzas a nivel mundial por no ser solo un deporte sino por formar parte de una actividad de salud. El cicloturismo es muy dinámico ya que se trata de poder hacer diferentes rutas en todas sus formas en cuanto a dificultad de terreno-distancia, puede ser tanto como un ciclopaseo por la ciudad, una ruta en carretera o descenso en una montaña. También es importante resaltar que un turista puede tanto hacer un viaje en bicicleta a larga distancia por varios días de manera independiente o también por contrato a una operadora de turismo local.

Dentro de las rutas ciclística están como propuestas las siguientes: Ruta City Bike tour, Ruta Ciclovía Bahía - Canoa, Ruta Bosque Cerro Seco, Ruta playa Chirije, Ruta La Fortuna y Ruta Las fragatas, cada una de estas como estrategia de reactivación turísticas y no solo como una actividad deportiva sino también recreativa e informativa.

\subsection{ESTRATEGIAS PARA GERENCIAR PRODUCTO TURISTICOS}

Según los resultados anteriores las estrategias que, desde criterio de experto, sobre estudios del estado del arte y sobre la técnica de observación realizada se pueden enunciar entre las principales estrategias las siguientes:

1. Estrategia de Posicionamiento: De acuerdo a las actividades según resultados de los instrumentos realizar estrategias de alianza con entidades públicas y privadas como auspiciantes para desarrollar actividades según atractivo turístico existente en la zona. 
2. Las estrategias de persuasión publicitarias: Realizar campañas publicitarias efectivas a través del aula creativa de la Extensión.

3. Estrategias de crecimiento en la entidad deportiva. Crear eventos deportivos como actividad de reactivación de acuerdo a la estacionalidad de la demanda anteriormente realizado.

4. Estrategia de Diversificación. Diversificar actividades deportivas y recreativas involucrando a los GAD de ambos cantones y auspiciantes locales, regionales y nacionales.

\section{CONCLUSION}

Los resultados de la encuesta aplicada y de la matriz aplicada por experto permitieron realizar un análisis correlativo donde demuestra que toda actividad deportiva se puede gerenciar como un producto turístico para fortalecer la sostenibilidad del turismo en el destino Sucre-san Vicente.

La existencia un total de 51 atractivos turísticos en el destino donde el $56 \%$ de estos atractivos los representan por su categoría sitios naturales demostrando que estas actividades deportivas podrán desarrollarse satisfactoriamente.

Fortalecer la actividad de ciclismo a partir de temporadas de acuerdo a la estacionalidad del turismo en la zona objeto estudio permitirá desarrollar varios productos turísticos en todo el destino y todo el año por sus especificaciones de ciclismo de montañas y ciclismo de rutas. 


\section{REFERENCIA BIBLIOGRÁFICA}

Arboleda, J. R. (s.f.). Facultad de Estudios Ambientales y Rurales -T.

Bohórquez, P. (2016). Proyecto de titulación previo a la obtención del título de Licenciada en Turismo, pág. 21

Clavijo. (2012). Repositorio UTE. Obtenido de Repositorio UTE: http://repositorio.ute.edu.ec/bitstream/123456789/1677/1/51585_1.pdf

Euromediterránea sobre Turismo y Desarrollo. (s.f.). Introducción al Concepto de Turismo Sostenible, pág. 2

Gallegos. (2007). Las Actividades Físico-Deportivas en la Naturaleza y la Industria Turística. Revista Internacional de Medicina y Ciencias de la Actividad Física y el Deporte vol. 7, pág. 111-127

INFORME JUVENTUD. (2010). Actividades Deportivas. Obtenido de https://www.zaragoza.es/contenidos/juventud/planjoven/15.pdf

López. (2004). POBLACIÓN MUESTRA Y MUESTREO. Punto Cero.

Martínez. (2004). Métodos de Muestreo. Ciencia UANL, pág. 121-123.

Middleton, M. y Marysela, M. C. (2004). Turismo y producto turístico. 150.

Morillo, M. (2011). Turismo y producto turístico. Evolución, conceptos, componentes y clasificación. Visión Gerencial, núm. 1, pàg.135-158.

Ojeda, C. D y Mármol Sinclair, P. (2012). Marketing Turístico. Madrid, España: Paranfino, S.A.

Oliviera, J. (1995). La crisis de la modernidad y el advenimiento de la posmodernidad: el deporte y las prácticas físicas alternativas en el tiempo de ocio activo. Apunts, Educación Física y Deportes, 10-29.

Peláez, M. E. (2009). El deporte como producto turístico. Revista Digital - Buenos Aires -, pag.137.

Pereira, A. L. (2003). Siglo XXI: nuevos valores, nuevas profesiones. Una perspectiva del ocio deportivo en la naturaleza integrado en el turismo. Lecturas Educación Física y Deportes, Revista Digital, pág. 3

Ramírez R. (2004). El impacto de la actividad física y el deporte sobre la salud, la cognición, la socialización y el rendimiento académico: una revisión teórica. Revista de Estudios Sociales SciElo.

SUCRE, G. (s.f.). EcuRed. Obtenido de EcuRed: https://www.ecured.cu/Cant\%C3\%B3n_Sucre_(Ecuador)

Turístico, S. (2017). EL SISTEMA TURÍSTICO SEGÚN ROBERTO BULLON. Obtenido de https://sistema-turistico.site123.me/teor\%C3\%8Das-del-sistema-tur\%C3\%8Dstico/el-sistematur\%C3\%8Dstico-seg\%C3\%9An-roberto-

bullon\#: :text=El\%20sistema\%20tur\%C3\%ADstico\%20es\%20concebido,de\%20uso\%20del\%20tiempo $\% 201 i b r e \%$ E2\%80\%9D. 
Unión Mundial para la Naturaleza, 1. (s.f.). Conceptos del Turismo Sostenible. Visión General del Turismo Sostenible, pág. 4. 